\title{
Whole blood cyanide levels in patients with tobacco amblyopia
}

\author{
JV JESTICO, MD O'BRIEN, R TEOH, PA TOSELAND, HC WONG \\ From the Departments of Neurology and Clinical Chemistry, Guy's Hospital, London, UK
}

SUMMARY Three patients presented with painless bilateral visual failure due to tobacco amblyopia. The whole blood cyanide levels were raised above those predicted from their high tobacco consumption, approaching lethal levels reported from acute inhalation of cyanide. Each patient had an excessive alcohol intake with biochemical evidence of hepatic dysfunction, the elevated whole blood cyanide levels being attributed to the associated impairment of cyanide detoxification. In each case the improvement in visual acuities following abstinence and hydroxycobalamin therapy was accompanied by a reduction in the whole blood cyanide level to within the normal range. Serial measurements of whole blood cyanide, serum alcohol, and the detection of urinary nicotine provided valuable indices of the patient's subsequent compliance and clinical progress.

Tobacco amblyopia presents as a progressive and painless bilateral visual failure. It characteristically affects elderly pipe smoking men, but has also been described in heavy cigarette smokers and in those who chew tobacco.' 2 Central vision is particularly impaired, with loss of colour perception and relative sparing of peripheral and night vision. Examination shows impaired visual acuity with centro-caecal scotomata. The fundoscopic appearances are usually normal, although temporal pallor of the optic discs may occur in longstanding cases. ${ }^{34}$

Pathological studies have described areas of demyelination in the optic nerves adjacent to the nerve head, affecting the papillo-macular bundle. ${ }^{5}$ In advanced cases the surrounding nerve fibres are also involved leading to destruction of the axis cylinders and secondary degeneration of the retinal ganglion cells. ${ }^{6}$

Patients with tobacco amblyopia have usually smoked heavily for many years before the onset of visual symptoms, and visual failure often develops with the occurrence of intercurrent disease. Many patients also drink heavily, and the condition has been designated tobacco/alcohol amblyopia. ${ }^{6-8}$ Two principle mechanisms have been proposed for its causation: firstly, nutritional deficiencies particularly of thiamine and vitamin $\mathrm{B} 12 ;^{6-10}$ and secondly, the

Address for reprint requests: Dr JV Jestico, Dept of Neurology, Guy's Hospital, St Thomas St, London SE1 9RT, UK.

Received 11 November 1983. Accepted 8 December 1983. toxic effect of the cyanide constituent of tobacco smoke." This report describes three cases of tobacco amblyopia to illustrate the usefulness of whole blood cyanide measurements and detection of urinary nicotine in the diagnosis of the condition and in subsequent patient management.

\section{Methods}

Cyanide measurements were made from $0.5 \mathrm{ml}$ of fresh whole blood taken from each patient using a modification of the method described by Rieders. ${ }^{12}$ A Cavett flask was used instead of the Conway vessel, providing a more airtight system. The cyanide present in each sample produces a purple dye in an alkaline medium by reaction with p-nitrobenzaldehyde and o-dinitrobenzine. This purple dye was then extracted at the end of the reaction and its absorbance measured at $555 \mathrm{~nm}$, to give a quantitative measure of the cyanide content of each sample. Very high cyanide levels were checked using the gas chromatographic method described by Valentour. ${ }^{13}$ The presence of nicotine in the urine was detected by thin layer chromatography following extraction with ether, using the method described by Jackson and Clatworthy. ${ }^{14}$

\section{Patients}

Case 1

A 55-year-old male plasterer presented with a 12 month history of progressive bilateral visual failure. For the first eight months he coped by borrowing spectacles from his workmates, but he then became unable to complete work sheets as he could no longer read. Distance vision was also impaired and approaching vehicles appeared as shadows. 
Table

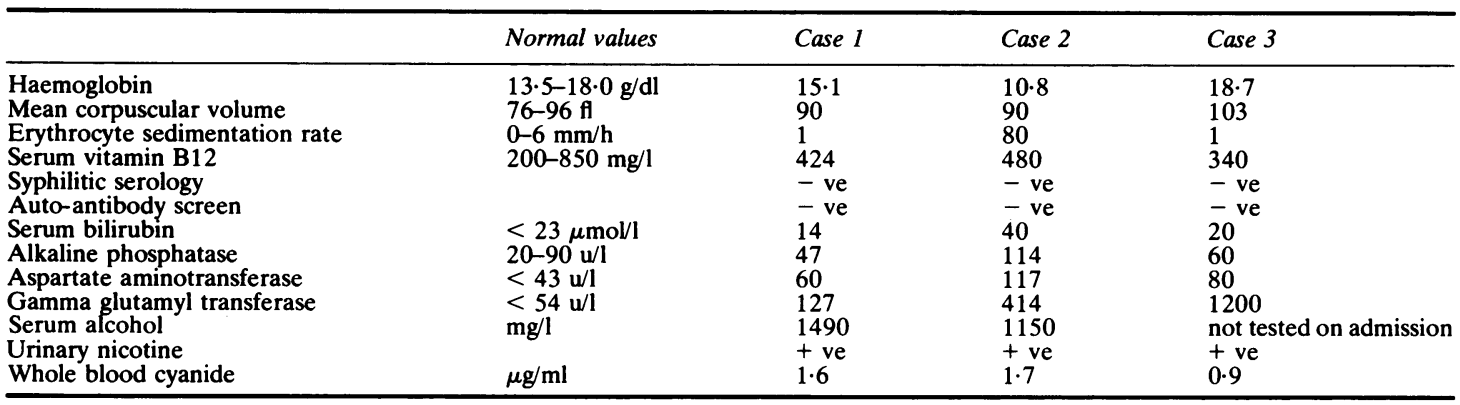

He became unable to recognise colours. He complained of morning frontal headaches, but was otherwise well. There was no significant past or family history. He smoked a pipe and hand rolled cigarettes containing pipe tobacco (CONDOR), consuming an ounce of tobacco per day. His daily alcohol intake was at least six pints of beer.

On examination the visual acuities were reduced to $6 / 60$ on each side. There were large bilateral central scotomata and a severe impairment of colour vision. The optic discs were pale. The pupils were large and barely reacted to light. There was a smooth enlargement of the liver $4 \mathrm{~cm}$ below the costal margin.

The relevant haematological and biochemical investigations are given in the table. A CT scan showed changes of cerebral atrophy. Visual evoked responses were of reduced amplitude, but normal latency.

A diagnosis of tobacco/alcohol amblyopia was made. The patient was urged to abstain from tobacco and alcohol and given a course of intramuscular hydroxycobalamin. His subsequent progress is shown in the figure. When reviewed two months later, his visual acuities had improved to $6 / 24$ on each side. He had stopped drinking alcohol, but was still smoking. He then claimed to have stopped smoking and four months later his acuities had further improved to $6 / 12$ (RE) and 6/18(LE). At this time the whole blood cyanide was $0.2 \mu \mathrm{g} / \mathrm{ml}$, the urinary nicotine being negative. Ten months later he was asymptomatic with visual acuities of 6/9 (RE) and 6/12 (LE) when he was discharged from follow up. Six months after this he returned complaining of a four week history of further painless visual deterioration. His marriage had broken up and he had lost his job. He was again drinking and smoking heavily. The visual acuities were reduced to $6 / 18$ on each side. The serum alcohol was $1930 \mathrm{mg} / \mathrm{l}$ and the whole blood cyanide $0.6 \mu \mathrm{g} / \mathrm{ml}$, the urinary nicotine being positive. He was again urged to abstain and given hydroxycobalamin. When last reviewed, three months later, he had improved with visual acuities of $6 / 12$ on each side, and the whole blood cyanide was less than $0 \cdot 1 \mu \mathrm{g} / \mathrm{ml}$, the urinary nicotine and serum alcohol being negative.

Case 2

A 56-year-old male time and motion expert presented with a four month history of progressive mistiness of vision in both eyes. He became unsafe when driving or crossing roads. He had to discontinue work as he was no longer able to compile time-tables, despite using a hand magnifying glass. He noticed that his vision was clearer in darkness than in daylight, and exposure to neon or bright lights induced frontal headaches. Five years previously he had had similar though less severe mistiness of vision. At that time his visual acuities were $6 / 9$ with normal visual fields, and prominent cupping of the optic discs was noted. No diagnosis was made at that time, and his symptoms resolved spontaneously after a period of two years. He had been a heavy pipe smoker since his teens, consuming two ounces of tobacco (CONDOR) per day. He admitted to drinking a bottle of whisky a day.

On examination the visual acuities were $6 / 24$ on each side. The visual fields were full to hand movements, but a bitemporal colour desaturation was demonstrated on confrontation with an $8 \mathrm{~mm}$ red pin. Goldman fields demonstrated bilateral centro-caecal scotomata. There was prominent optic disc cupping, but no other fundoscopic abnormality. He was plethoric and jaundiced, with spider naevi over his chest. The liver was palpable $6 \mathrm{~cm}$ and the spleen 3 $\mathrm{cm}$ below the costal margins.

Relevant haematological and biochemical investigations are given in the table. Radiology of the skull with coned views of the pituitary fossa and orbital foramena were normal as was a cerebral CT scan with orbital views. The visual evoked responses were of small amplitude but normal latency. Barium swallow showed extensive oesophageal varicies. A liver biopsy showed changes of cirrhosis with fibrosis, cellular infiltration and some fatty change.

A diagnosis of tobacco amblyopia was made, and treatment instituted with intramuscular hydroxycobalamin. He was urged to abstain from tobacco and alcohol. Three days after starting treatment he was sent home on weekend leave. On his return the serum alcohol and urinary nicotine were negative. When reviewed one month later his visual acuity had improved to $6 / 9$ on each side and the whole blood cyanide, urinary nicotine and serum alcohol estimations were negative. These estimations remained negative during the following six months, the acuities being 6/6 (RE) and 6/9 (LE). He then started smoking and drinking again, though claiming to consume less than before. He had no further visual symptoms, but had three emergency admissions with bleeding oesophageal varicies. When seen after this his visual acuities were unchanged, the whole blood cyanide was $0.2 \mu \mathrm{g} / \mathrm{ml}$ and the serum alcohol was not detectable. 

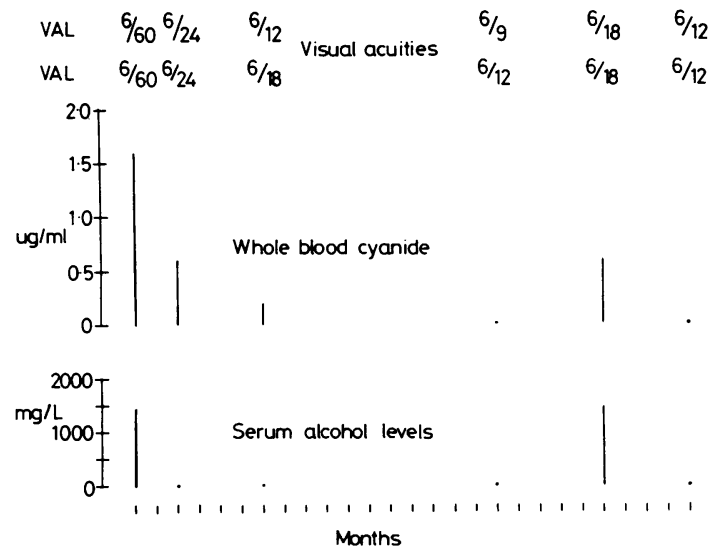

Fig Changes in visual acuity, whole blood cyanide and serum alcohol levels of a 55-year-old man (Case 1) with tobacco amblyopia (see text). Above: Visual acuities, right and left eyes respectively. Middle Graph: Whole blood cyanide levels ( $\mathrm{g} / \mathrm{ml})$. Lower Graph: Serum alcohol levels $(m g / l)$.

\section{Case 3}

A 42-year-old unemployed baker had a congenital squint and his early educational difficulties had been attributed to his amblyopic right eye. At the age of 9 years the squint was corrected surgically. Since childhood he had required spectacles for reading and close work, but his vision remained unchanged until he presented with a three year history of slowly progressive visual failure. Despite new spectacles he became unable to read. He found occasional employment as a "minicab" driver, but was forced to discontinue this as he was no longer able to recognise road signs. On-coming vehicles appeared only as vague outlines at a distance of 18 metres, and he was unable to identify their colours. He smoked 40 "full strength" cigarettes (CAPSTAN) and drank two bottles of sherry per day.

On admission the visual acuities were 6/60 (RE) and $6 / 36$ (LE); he had a gross impairment of colour vision. There were bilateral central scotomata. The right optic disc was pale, the left appeared normal. The reflexes were symmetrical with flexor plantar responses. Vibration sensibility was lost below the iliac crests, and pain and tactile sensation were impaired in a glove and stocking distribution suggesting a peripheral neuropathy.

The relevant haematological and biochemical investigations are given in the table. Cerebral CT scan including orbital views was normal. Nerve conduction studies were normal. Visual evoked responses were small, but with normal latency.

He was urged to abstain from both tobacco and alcohol and treated with hydroxycobalamin. When last seen one month after discharge, the visual acuities had improved to $6 / 18$ on each side.

\section{Discussion}

All three of our patients with tobacco amblyopia had excessive alcohol intake. The frequent associa- tion of such amblyopia with chronic alcoholism has led previous authors to conclude that nutritional deficiences rather than the effects of toxic substances are responsible for the optic nerve damage in tobacco amblyopia. ${ }^{78}$ However, not all patients with tobacco amblyopia are alcoholics, alcoholism occurring in about two thirds of patients. ${ }^{3}$ The role of nutritional deficiencies has been investigated by studies of hospitalised patients with tobacco amblyopia who were allowed to continue smoking and drinking. These studies have described improvement in visual acuities with reduction in the size of centro-caecal scotomata following the administration of dietary supplements, namely brewer's yeast and B vitamins. ${ }^{571516}$ However, it is likely that their consumption of alcohol and tobacco was reduced while in hospital. Further evidence for the role of nutritional deficiencies has come from the studies of prisoners of war, describing visual failure identical to that of tobacco amblyopia occurring in those deprived of both tobacco and alcohol. ${ }^{17}{ }^{18}$ Each of our cases had been assessed by the dietician, and it was considered that their dietary intake was adequate, and as such we concluded that there was no evidence that nutritional deficiencies were responsible for their visual failure.

The vitamin B12 estimations in our patients were all within normal range. However, low serum vitamin B12 levels have been reported to occur in $30 \%$ of patients with tobacco amblyopia, ${ }^{9}$ and methylmelanuria, a further index of vitamin B12 deficiency has also been described in such patients. ${ }^{19}$ Because of these observations, vitamin B12 injections, in the form of hydroxycobalamin, together with abstinence has become the standard therapy for tobacco amblyopia and each of our patients received a course of hydroxycobalamin.

The centro-caecal scotomata are the characteristic visual field defects seen in patients with toxic amblyopias. They reflect damage to the optic nerve fibres constituting the maculo-papillar bundle, which are particularly sensitive to the effects of toxic substances. ${ }^{20}$ That such scotomata are the characteristic field defects found in patients with tobacco amblyopia, perhaps provides indirect clinical evidence that the condition is the result of some exogenous toxin. It has been suggested that the cyanide constituent to tobacco smoke acts as such a toxin in the causation of visual failure in patients with tobacco amblyopia." In support of this, symmetrical demyelinating lesions of the retro-bulbar parts of the optic nerves have been produced experimentally in animals by chronic exposure to sodium cyanide. ${ }^{21}$

The principal method of cyanide detoxification involves the enzymes rodalase ${ }^{22}$ and mercaptopyru- 
vate sulphurtransferase, present in the liver, kidneys and gastrointestinal tract. ${ }^{23}$ By these, cyanide is converted to carbon dioxide and thiocyanate, which is non-toxic and excreted in the urine. Hydroxycobalamin is also considered to play a role in cyanide detoxification by combining with the cyanide radicals to produce the non-toxic cyanocobalamin. ${ }^{24}$ Indeed the administration of high doses of hydroxycobalamin has been shown to protect animals from otherwise lethal doses of cyanide. ${ }^{25}$ In addition, in patients with tobacco amblyopia, it has been shown that hydroxycobalamin is more effective than cyanocobalamin in the treatment of the condition. ${ }^{26}$

Serum thiocyanate measurements have been widely used as an index of a patient's smoking habits, and have been reported to closely follow their tobacco consumption. ${ }^{27}$ However, studies of patients with tobacco amblyopia have drawn attention to the fact that the serum thiocyanate levels may be surprisingly low compared with the levels predicted from their tobacco consumption. ${ }^{28}$ This has suggested an impairment of cyanide detoxification in patients with tobacco amblyopia, again implying that cyanide is the toxic substance responsible for the condition. Furthermore following the administration of hydroxycobalamin the serum and urinary thiocyanate levels increase, suggesting an improvement in cyanide detoxification. ${ }^{29}$

Measurement of the serum thiocyanate, as an index of cyanide metabolism has been advocated on the grounds of its stability. ${ }^{30}$ However, this may not always provide a true reflection of a patient's smoking habits. This is because the thiocyanate metabolic pool is normally large and may be greatly influenced by variations in dietary intake of pre-formed thiocyanate and plant cyanogenic glycosides, in particular onions. ${ }^{31}$ Also, thiocyanate levels may be affected by even minor degrees of dehydration particularly with vomiting. ${ }^{32}$ Thus misleading results may occur. In patients with tobacco amblyopia the measurement of the whole blood cyanide has advantages over measurement of serum thiocyanate. Firstly, it is not influenced by extraneous dietary intake. Secondly it provides a direct measure of the toxic substance considered to be responsible for the condition, as opposed to an indirect measure of its detoxification products. The disadvantage of whole blood cyanide measurements is that the blood specimen must be analysed immediately.

Exposure to hydrogen cyanide gas is recognised as one of the principal causes of death in domestic fires, the gas being liberated from burning polyurethane foam present in many household furnishings. Post mortem whole blood cyanide levels in victims of house fires have been reported as being between $0 \cdot 2$ $\mu \mathrm{g} / \mathrm{ml}$ and $2 \cdot 2 \mu \overline{\mathrm{g}} / \mathrm{ml}{ }^{33}$ Cyanide levels between $2 \cdot 2$ $\mu \mathrm{g} / \mathrm{ml}$ and $2.6 \mu \mathrm{g} / \mathrm{ml}$ have also been found in fatal cases (Toseland, unpublished data). Accurate determination of whole blood cyanide necessitates that the blood samples be delivered as quickly as possible to the laboratory, from the necropsy room. Because this is not always possible it is suspected that the lethal level of whole blood cyanide may be considerably higher at the time of death. ${ }^{34}$ High blood cyanide levels may be recorded from the patients receiving sodium nitroprusside in diuretic therapy. In one instance a whole blood cyanide of $6 \cdot 1 \mu \mathrm{g} / \mathrm{ml}$ was recorded from a patient receiving sodium nitroprusside, who subsequently recovered. In other patients on nitroprusside therapy, whole blood cyanide levels have been below $1.2 \mu \mathrm{g} / \mathrm{ml}$ (Toseland, unpublished data).

The cyanide concentration in tobacco smoke may be very high, for example up to 1500 parts per million. ${ }^{35}$ The whole blood cyanide level in nonsmokers is usually less than $0 \cdot 1 \mu \mathrm{g} / \mathrm{ml}$. However, in the case of heavy smokers, the whole blood cyanide may be up to $0.3 \mu \mathrm{g} / \mathrm{ml} .^{36}$ It was, therefore, of particular interest to find much higher levels of whole blood cyanide in each of our three patients with tobacco amblyopia, implying that such chronic exposure to tobacco smoke can result in extremely high levels of cyanide without ill effects other than amblyopia, despite such levels approaching the lethal range for acute exposure to the toxin.

The whole blood cyanide levels of our three patients with tobacco amblyopia were considerably higher than those expected from non-amblyopic subjects with a similar high tobacco consumption. Such high levels suggest an impairment of cyanide metabolism in these patients. As recognised by previous reports, an excessive alcohol intake is the most obvious association with the disorder. Two of our patients had hepatic enlargment, one having obvious liver failure, and all three having biochemical evidence of hepatic insufficiency. We therefore concluded that without additional metabolic abnormalities, exposure to high concentrations of cyanide present in tobacco smoke may be tolerated. However, with the occurrence of an impairment of cyanide detoxification, in our cases in relation to hepatic dysfunction, the whole blood cyanide may increase considerably, and such chronic accumulation of cyanide is a principal factor responsible for the causation of tobacco amblyopia.

The cases reported in this study illustrate how useful whole blood cyanide measurements can be in the diagnosis of tobacco amblyopia. In our three patients we considered the findings of high cyanide levels, above those predicted from their tobacco consumptions, to be diagnostic of the condition. The 
serial measurements of the whole blood cyanide provided an extremely useful method of monitoring the subsequent clinical course of our patients with tobacco amblyopia. In each case, the improvement of visual acuity following treatment was accompanied by a fall in the whole blood cyanide levels, eventually to within the normal range. The usefulness of such serial measurements was shown in our first case, who relapsed following his marital breakdown, when he resumed smoking and drinking heavily, and the cyanide levels increased.

After smoking cigarettes, nicotine can be detected in the urine for up to 24 hours (Toseland, unpublished data). Because of this, the simple detection of urinary nicotine provides a valuable index as to whether a patient is still smoking. This was illustrated by the negative result obtained in our second patient on his return from weekend leave, confirming that he had indeed abstained from smoking. As with alcoholics, patients with tobacco amblyopia may be reluctant to disclose whether or not they have actually stopped smoking, or what their actual tobacco consumption really is. Clearly these patients require careful follow up and repeated encouragement to abstain, and we consider that repeated measurements of the whole blood cyanide, serum alcohol, and the detection of urinary nicotine, provide accurate indices of a patient's compliance and tobacco consumption, which will hopefully lead to better management of the condition.

\section{References}

${ }^{1}$ Beer GJ. Lehre Von Den Augenkrankheiten Vol 2. Vienna, 1817.

2 Traquair HM. Toxic amblyopias including retrobulbar neuritis. Trans Ophthalmol Soc UK 1930;50:315-85.

${ }^{3}$ Foulds WS, Pettigrew AR. The Biochemical Basis of the Toxic Amblyopias. In: Perkins ES, Hill DM, eds. Scientific Foundations of Ophthalmology. Heineman Press, 1977:50-4.

${ }^{4}$ Dreyfus PM. Amblyopia and other Neurological Disorders Associated with Chronic Alcoholsim. In: Vinken PJ, Bruyn GW, eds. Handbook of Clinical Neurology. Amsterdam: North Holland Publishing Co. 1976;28:331-47.

5 Victor M, Mancall EL, Dreyfus PM. Deficiency amblyopia in the alcoholic patient. Arch Ophthalmol 1960;64: 1-33.

- Victor M, Dreyfus PM. Tobacco-alcohol amblyopia. Arch Ophthalmol 1965;74:649-57.

7 Carroll FD. Aetiology and treatment of tobacco-alcohol amblyopia. Am J Ophthalmol 1944;27:847-63.

${ }^{8}$ Victor M. Tobacco-alcohol amblyopia. A critique of current concepts of this disorder with special reference to the role of nutritional deficiency and its causation. Arch Ophthalmol 1963;70:313-7.
${ }^{9}$ Heaton JM, McCormick AJA, Freeman AG. Tobacco amblyopia-a clinical manifestation of vitamin B12 deficiency. Lancet 1958;2:286-90.

${ }^{10}$ Potts AM. Tobacco amblyopia. Surv Ophthalmol 1973;17:313-39.

"Wokes F. Tobacco amblyopia. Lancet 1958;2:526-7.

${ }^{12}$ Rieders F. In: Sunshine I, ed: Methodology for Analytical Toxicology. Cleveland: The Chemical Rubber Co. 1975:113-5.

${ }^{13}$ Valentour JC, Aggarwal V, Sunshine I. Sensitive gas chromatographic determination of cyanide. Ann Chem 1974;46:924-7.

14 Jackson JV, Clatworthy AJ. Toxicological Applications of Chromatography. In: Smith I, Seakins JWT, eds. Chromatographic and Electrophoretic Techniques 1976;1:380-453.

15 Carroll FD. Recurrence of tobacco-alcohol amblyopia. Am J Ophthalmol 1945;28:636-9.

${ }^{16}$ Carroll FD. Nutritional retrobulbar neuritis. Am J Ophthalmol 1947;30:172-6.

${ }^{17}$ Smith DA, Woodruff MFA. Deficiency Disease in Japanese Prison Camps. Medical Research Council, Special Services Report Series. London: Her Majesty's Stationery Office No 274 (1951).

${ }^{18}$ Fisher CM. Residual neuropathological changes in Canadians held prisoners of war by the Japanese. Can Serv Med J 1955;11:157-99.

${ }^{19}$ Heaton JM. Methylmalonic acid excretion and tobacco amblyopia. Lancet 1963;2:789-90.

${ }^{20}$ Glaser JS. (ed) In: Neuro-Ophthalmology. Hagerstown, Maryland: Harper and Row, 1978:12.

${ }^{21}$ Lessel S. Experimental cyanide optic neuropathy. Arch Ophthalmol 1971;84:194-204.

${ }^{22}$ Lang K. Die Rhodambildung im Tierkörper. Biochem $Z$ 1933;259:243-56.

${ }^{23}$ Fiedler H, Wood JL. Specificity studies on the B-Mercaptopyruvate-Cyanide Transulphuration system. J Biol Chem 1956;222:387-97.

${ }^{24}$ Smith ADM. Retrobulbar neuritis in Addisonan penicious anaemia. Lancet 1961;1:1001-2.

${ }^{25}$ Boxer GE, Rickards JC. Studies of the metabolism of the carbon of cyanide and thiocyanate. Arch Biochem 1952;39:7-26.

${ }^{26}$ Chisholm IA, Bronte-Stewart J, Foulds WS. Hydroxycobalamin versus cyanocobalamin in the treatment of tobacco amblyopia. Lancet 1967;2:450-1.

${ }^{27}$ Støa KF. Studies on thiocyanate in serum with some supplementary investigation in saliva, urine and cerebrospinal fluid. Arbok Univ Bergen Med 1956-1957, Ser. 2; 1957:1-165.

${ }^{28}$ Chisholm IA, Pettigrew AR. Biochemical observations in toxic optic neuropathy. Trans Ophthalmol Soc UK 1970;90:827.

${ }^{29}$ Foulds WS, Bronte-Stewart JM, Chisholm IA. Serum thiocyanate concentrations in tobacco amblyopia. Nature 1968;218:586.

${ }^{30}$ Pettigrew AR, Fell GS. Microdiffusion method for estimation of cyanide in whole blood and its application to the study of conversion of cyanide to thiocyanate. Clin Chem 1973;19:466-71.

${ }^{31}$ Montgomery RD. Cyanogens. In: Liener IE, ed. Toxic Constituents of Plant Food Stuffs. New York: 
Academic Press, 1969:143-57.

${ }^{32}$ Montgomery RD. Cyanogenic Glycosides. In: Vinken PJ, Bruyn GW, eds. Handbook of Clinical Neurology. Amsterdam: North Holland Publishing Co. 1979:515-27.

${ }^{33}$ Wetherell HR. The occurrence of cyanide in the blood of fire victims. J Forens Sci 1966;11:167-73.

${ }^{34}$ Curry AS. Cyanide poisoning. Acta Pharmacol Toxicol
1963;20:291-4.

${ }^{35}$ U.S. Department of Health, Education and Welfare, Public Health Service 1964. Smoking and Health. Report of the Advisory Committee to the Surgeon General. Public Health Service Publication No 1103.

${ }^{36}$ Clark CJ, Campbell D, Reid WH. Blood carboxyhaemoglobin and cyanide levels in fire survivors. Lancet 1981;1:1332-5. 